\title{
Evaluación de la capacidad de sellado de MTA, IV y OZE en retrobturación apical.
}

\section{Evaluation of the sealing capacity of MTA, IV and OZE in apical retrobturation.}

\author{
Dones Valentina ${ }^{1}$, Mohn Claudia E $E^{2}$,Gualtieri Ariel ${ }^{3}$,Pinasco Laura E E $^{1}$, Sierra Liliana G ${ }^{1}$,Rodriguez Pablo \\ $\mathbf{A}^{1 *}$,Casadoumecq Ana Clara ${ }^{1}$ \\ ${ }^{1}$ Cátedra de Endodoncia, Facultad de Odontología, Universidad de Buenos Aires.Argentina. ${ }^{2}$ Cátedra de Fisiología, \\ Facultad de Odontología, Universidad de Buenos Aires.Argentina. ${ }^{3}$ Cátedra de Biofísica, Facultad de Odontología, \\ Universidad de Buenos Aires.Argentina. \\ * prodriguez@odontología.uba.ar
}

\begin{abstract}
Resumen
En el presente trabajo, se evaluó la capacidad de sellado del mineral de trióxido agregado (MTA), ionómero vitreo (IV) y oxido zinc eugenol (OZE) en retrobturaciones apicales. Cien piezas dentales unirradiculares, fueron divididas en 5 grupos $(\mathrm{n}=20)$ : 1- control positivo, ápice sin sellar; 2-control negativo, ápice impermeabilizado con esmalte de uñas; 3- retrobturado con MTA; 4- retrobturado con OZE; 5- retrobturado con IV. Al finalizar los tratamientos ex vivo, las piezas fueron sometidas a la prueba de penetración de tinte, mediante sumersión en azul de metileno $2 \%$ durante 48 hs. A continuación, se realizó un corte transversal a $1 \mathrm{~mm}$ del extremo apical y se midió el porcentaje de tinción como medida de la capacidad de sellado de los materiales de obturación empleados. Los resultados fueron analizados con la prueba de Kruskal-Wallis, seguida por comparaciones de a pares. El porcentaje de tinción no difirió significativamente entre los tratamientos con MTA e IV, pero éstos mostraron un porcentaje significativamente menor con respecto al tratamiento con OZE. Además, no se encontraron diferencias significativas entre el tratamiento con OZE y el control positivo. Estos resultados demuestran que el IV y el MTA tienen similar capacidad de sellado en retrobturación in vitro, y que a su vez, constituyen mejores selladores que el OZE, que no demostró ser un sellador efectivo, al menos en las condiciones estudiadas.
\end{abstract}

Palabras clave: Obturación Retrógrada, mineral de trióxido agregado, ionómero vitreo, oxido zinc eugenol.

\begin{abstract}
In the present work, the sealing capacity of the aggregate trioxide ore (MTA), vitreous ionomer (IV) and zinc oxide eugenol $(O Z E)$ in apical retrobturations was evaluated. One hundred unirradicular dental pieces were divided into 5 groups $(n=$ 20): 1- positive control, unsealed apex; 2-negative control, apex waterproofed with nail polish; 3- retrobturado with MTA; 4- retrobturado with OZE; 5-retrobturado with IV. At the end of the ex vivo treatments, the pieces were subjected to the dye penetration test, by submerging in $2 \%$ methylene blue for 48 hours. Next, a cross section was made $1 \mathrm{~mm}$ from the apical end and the percentage of staining was measured as a measure of the sealing capacity of the sealing materials used. The results were analyzed with the Kruskal-Wallis test, followed by pairwise comparisons. The percentage of staining did not differ significantly between treatments with MTA and IV, but these showed a significantly lower percentage compared to treatment with OZE. In addition, no significant differences were found between the treatment with OZE and the positive control. These results demonstrate that IV and MTA have similar sealing capacity in retrobturation in vitro, and that in turn they constitute better sealants than OZE, which did not prove to be an effective sealant, at least under the conditions studied.
\end{abstract}

Key words: Retrograde filling, aggregate trioxide ore, vitreous ionomer, eugenol zinc oxide.

\section{INTRODUCCIÓN}

La endodoncia quirúrgica está indicada cuando el tratamiento endodóntico no quirúrgico fracasa. Incluye resección apical, preparación retrocavitaria y retrobturación apical. ${ }^{1}$ Una cavidad de clase I de 3 milímetro $(\mathrm{mm})$ de profundidad preparada con instrumental ultrasónico deberá ser obturada con un material que garantice un sellado hermético.

El objetivo principal de la retrobturación apical es evitar la difusión de las bacterias y sus productos desde el canal radicular a los tejidos periapicales y viceversa. El sellado retrogrado se realiza para minimizar los espacios y mejorar la calidad de la obturación. Las pruebas de penetración de 
tinte son un método sencillo y útil en la evaluación de los materiales de sellado. Si los materiales son capaces de evitar la filtración de pequeñas moléculas, potencialmente, pueden impedir la filtración de sustancias más grandes, tales como bacterias y sus productos. ${ }^{2,3}$ Gartner y Dorn propusieron que un material de obturación del ápice ideal debería ser, fácil de manipular, radiopaco, dimensionalmente estable, no absorbible, insensible a la humedad, adhesivo a la dentina, no tóxico y biocompatible. ${ }^{4}$ Muchos materiales son utilizados con este fin pero ninguno es universalmente aceptado como el mejor. Se han sugerido: gutapercha, amalgama, óxido de zinc eugenol (OZE), Cavit, resina compuesta, láminas de oro y ionómeros vítreos (IV), entre otros. ${ }^{4-6}$

La amalgama ha sido el material más extensamente utilizado. Sin embargo, en la década pasada la amalgama cedió lentamente su lugar a los materiales conteniendo OZE, tales como el IRM y el SuperEBA [cemento de óxido de zinc reforzado basado en una mezcla de $32 \%$ de eugenol y $68 \%$ de ácido ortoetoxibenzoico (EBA)], como los materiales favoritos de obturación apical. Numerosos estudios demostraron que los materiales conteniendo OZE son superiores a la amalgama en términos de sellado y biocompatibilidad. ${ }^{7,8}$ Más recientemente, y gracias a sus propiedades, el mineral de trióxido agregado (MTA) ha sido sugerido como el mejor material de obturación apical. Los principales componentes del MTA son el silicato tricálcico (Ca3Si), el aluminato tricálcico $(\mathrm{Ca} 3 \mathrm{Al})$ y el óxido tricálcico $(\mathrm{Ca} 3 \mathrm{O} 2)$. Debido a su mayor capacidad de sellado, por sobre los materiales de obturación convencionales, y a su biocompatibilidad, el MTA está ganando popularidad entre los endodoncistas. ${ }^{9,10}$ Estudios in vivo han demostrado que el MTA posee la capacidad de inducir la formación de hueso, dentina y cemento. ${ }^{11,12}$ En un estudio que evalúa las propiedades inductivas del MTA sobre hueso y dentina, se demostró que los cultivos celulares de osteoblastos y dentinoblastos maduros crecen mejor y más rápidos en medios con MTA que en medios sin el compuesto. ${ }^{13}$ Puede ser prematuro asegurar que el MTA es el material de obturación apical ideal, sin embargo, los resultados de los experimentos llevados a cabo usando modelos animales y métodos de laboratorio in vitro, conjuntamente con métodos celulares y moleculares modernos, proveen evidencia de que el MTA posee un gran potencial inductor de la curación, y es más biocompatible que muchos otros materiales de retrobturación apical disponibles en la actualidad. ${ }^{11-14}$ Algunos resultados clínicos experimentales también concuerdan con estos resultados obtenidos de experimentos in vitro o a partir de estudios realizados en animales (10). Sin embargo, el uso del MTA por sí solo no garantiza el éxito clínico, así como tampoco puede superar las deficiencias de técnicas que son inherentes a la preparación apical tradicional. Estudios clínicos han demostrado que la obturación con MTA mejora la curación de pacientes con respecto a pacientes obturados sin el compuesto. ${ }^{15}$ Por su parte, el IV es indicado en endodoncia para el sellado de perforaciones y defectos de reabsorción de la raíz, el tratamiento de las fracturas verticales, la obturación retrograda y el sellado coronal. El IV tiene la capacidad de unirse a los tejidos dentarios, especialmente a la dentina, es biocompatible y libera flúor a largo plazo. Estas cualidades hacen que su uso sea ventajoso en endodoncia y en la odontología restauradora, siendo uno de los materiales incluidos en el protocolo de cirugía apical de la Cátedra de Endodoncia de la Facultad de Odontología de la Universidad de Buenos Aires (FOUBA). A pesar de las características críticas de su manejo clínico, el IV ha mostrado resultados concluyentes con respecto a su capacidad de sellado y actividad antimicrobiana, y existe evidencia sustancial para confirmar su rendimiento clínico satisfactorio. Además, su compatibilidad con los tejidos duros y blandos lo hacen adecuado para su uso en cirugía endodóntica. La microfiltración es la causa más común responsable del fracaso del tratamiento endodóntico. Se han empleado una variedad de métodos para evaluar la capacidad de retrobturación apical tal como la penetración de radioisótopos, el grado de penetración del tinte, las técnicas de filtración de líquidos, los métodos electroquímicos, la penetración bacteriana, la microscopía electrónica de barrido y la porometría de flujo capilar. El método de penetración de tinte utilizado para medir la capacidad de sellado es el más popular. Varios tintes que se pueden usar incluyen tinta India, fucsina básica, nitrato de plata, azul de metileno y rodamina B. ${ }^{15}$ Aunque muchas soluciones diferentes han sido utilizado en estudios de investigación para evaluar filtraciones, el azul de metileno ha sido el más utilizado para evaluar la penetración del tinte como medida para cuantificar microfiltración ${ }^{16,17}$ Por todo lo expuesto, el objetivo del presente estudio fue comparar la capacidad de sellado de MTA, IV y OZE in vitro como materiales de retrobturación en cirugías apicales.

\section{MATERIALES Y MÉTODOS}

Se tomaron 100 piezas dentales unirradiculares extraídas de hombres y mujeres (18 y 25 años de edad) por motivos ortodoncicos. Los criterios para la selección de los dientes incluyeron una sola raíz sin curvatura, sin caries, fractura o grietas. Se lavaron, se desbridaron con $\mathrm{NaOCl}$ al $5 \%$ y luego se almacenaron en solución salina normal hasta su uso. Las coronas se seccionaron en la unión cemento-esmalte usando un disco de diamante bajo riego constante con agua a una longitud estandarizada de $15 \mathrm{~mm}$.

Se trataron con técnica manual apico-coronal, utilizando fresas de gates-glidden (N 3, 2 y 1) y luego limas K (Maileffer, Dentsply). Se intrumentó hasta el calibre 40, 0,5 mm por antes de la longitud de trabajo y el resto del canal se ensanchó utilizando la técnica de step-back hasta calibre 50. Se irrigó con hipoclorito de sodio, y se secó con conos de papel absorbente. Se obturaron con técnica de compactación lateral con conos de gutapercha y cemento de Grossman (Radi-fill, Dickinson). Para sellar la cámara pulpar se grabaron las paredes con ácido fosfórico, se aplicó adhesivo 
(Adper Single Bond,3M ESPE, USA) y luego se obturó con resina fotopolimerizable (filtek Z350 XT, 3M ESPE, USA), incluyendo en cada una de ellas un hilo de tanza para luego poder colgar cada pieza dental de una vara. Se sumergieron todas las piezas dentales (solo la raíz hasta el límite amelocementario) en esmalte de uñas ${ }^{3,18,19}$ (Colorama, São Paulo, SP, Brazil) 3 veces consecutivas con un intervalo de secado de 30 minutos entre cada capa para impermeabilizarlas. Se tomó cada pieza dental y se la colocó en una morsa para fijarla y así poder realizar la resección apical. Estudios anatómicos del ápice radicular muestran que al menos $3 \mathrm{~mm}$ del ápice deben ser resecados a fin de reducir el $98 \%$ de las ramificaciones apicales y el $93 \%$ de los conductos laterales. ${ }^{20}$ Se procedió a amputar $3 \mathrm{~mm}$ apicales de cada pieza de toda la muestra con bisturí piezoeléctrico (Variosurg, NSK, Japón), mediante un corte perpendicular al eje mayor de la raíz. Se retroprepararon los conductos radiculares mediante un retrotip ultrasónico (AS3D - Satelac) haciendo un ligero movimiento de cepillado (profundidad de $3 \mathrm{~mm}$ ). Se los dividió en 5 grupos utilizando la aplicación para celular Random Number Generator Plus Dice, Lotto, Coins. Se los retroobturó con diferentes materiales según le corresponda a cada grupo: Grupo 1- control positivo $(n=20)$ : se dejaron con el ápice sin sellar. Grupo 2control negativo $(n=20)$ : ápice impermeabilizado con esmalte de uñas. Grupo 3- MTA $(n=20)$ : retrobturado con MTA (ProRoot $R$ MTA; Dentsply Tulsa Dental, Tulsa, OK). Se preparó el MTA mezclando el polvo con agua estéril en una proporción de 3:1 con una espátula metálica sobre una loseta estéril (10). Con un condensador quirúrgico, se obturaron las superficies radiculares y se lo dejo fraguar en presencia de una torunda estéril húmeda dado que requiere humedad para fraguar. La hidratación del MTA resulta en un gel coloidal que solidifica en un lapso de 3 a 4 horas, según indicación del fabricante. ${ }^{21}$ Grupo 4- OZE $(n=20)$ : retrobturado con OZE (IRM, Dentsply). Se mezclaron el polvo y su solvente líquido con espátula metálica sobre una loseta de vidrio y se colocó el cemento en la preparación dentaria con un condensador quirúrgico. Se esperó su endurecimiento. Tiempo inicial de fraguado se produce a los 5 minutos de iniciada la mezcla, según indicación del fabricante. Grupo 5- IV (n=20): retrobturado con IV (Fuji 9, GC, Japón). Se mezcló sobre un pad de papel una parte de polvo y una gota del solvente líquido con una espátula plástica. Se colocó el cemento sobre la preparación dentaria no desecada y se esperó el tiempo de fraguado. Tiempo de trabajo 2 minutos. Tiempo inicial de fraguado se produce a los 6 minutos, según indicación del fabricante. Las muestras se almacenaron en frascos de vidrio con algodones humedecidos y al calor de una lámpara que los mantuvo a una temperatura de $37 \mathrm{C}$ durante 24 horas. A continuación, las 100 piezas se prepararon para la tinción, colgándolas de una vara de madera dentro de un recipiente plástico. Se las niveló para que todas estuvieran a la misma altura y sin tocar el fondo del recipiente. Se las sumergió en el colorante azul de metileno al $2 \%$ por 48 horas a temperatura ambiente..$^{2,22}$ A continuación, se lavaron durante 30 minutos con agua corriente. Una vez secas, se las incluyó en acrílico cristal de autocurado logrando unos tacos cilíndricos para poder proceder a realizar los cortes. A cada una de las piezas de toda la muestra se le realizó 1 corte transversal a una distancia de $1 \mathrm{~mm}$ desde el borde apical de la pieza con un disco de diamante refrigerado a baja velocidad ${ }^{23,24}$ en la cátedra de materiales dentales (FOUBA). Los cortes fueron realizados por la misma operadora con regla milimetrada marcando en las 4 caras marcas previas guía para controlar el corte. Las imágenes de los cortes fueron digitalizadas y evaluadas mediante el software Ip Wip 32 que mide el área total de la pieza dental y el área coloreada con azul de metileno. Luego se calculó el porcentaje de tinción de cada muestra.

Análisis estadístico: Se comparó el porcentaje de tinción entre los distintos tratamientos utilizando la prueba no paramétrica de Kruskal-Wallis, seguida por comparaciones de a pares. Se fijó un nivel de significación del $5 \%$ (alfa = 0.05). Para cada muestra se obtuvieron los siguientes parámetros descriptivos de la variable "porcentaje de tinción": media, desviación estándar (DE), mínimo (Mín.), máximo (Máx.), mediana, primer cuartil (Q1) y tercer cuartil (Q3). La descripción de los resultados se complementó con un gráfico de cajas, que presenta las siguientes características: los extremos inferior y superior de cada caja corresponden a mínimo y máximo, respectivamente; los bordes inferior y superior, a Q1 y Q3, respectivamente; la línea interna de la caja corresponde a la mediana; el punto indica la posición de la media. Una caja "incompleta", en donde no se observan todos los elementos gráficos mencionados anteriormente, indica que diferentes parámetros presentan el mismo valor. Se utilizó el programa Infostat versión 2013 p. ${ }^{25}$

\section{RESULTADOS}

Una vez realizados los cortes se analizaron las superficies dentarias teñidas:

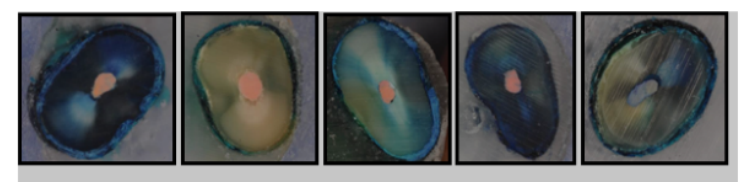

Fig. 1. Control positivo, Control negativo, MTA, OZE, IV.

Se obtuvo de cada muestra el porcentaje (proporción de superficie teñida sobre el total de la superficie transversal) que se había teñido con el colorante y se confeccionó una tabla.

Se detectó una diferencia significativa global del porcentaje de tinción entre los grupos comparados (prueba de Kruskal-Wallis: $\mathrm{H}=52.09 ; \mathrm{gl}=4 ; \mathrm{p}<0.05$ ). Este resultado global indica que existen diferencias significativas entre al 
menos dos grupos. Para conocer específicamente entre qué pares de grupos hay diferencias significativas, se realizan comparaciones de a pares post hoc, cuyos resultados se visualizan en la en la Tabla 1 y en la Figura 2. El porcentaje de tinción no difirió significativamente entre los tratamientos con MTA y IV, pero estos dos tratamientos mostraron diferencias significativas con respecto al tratamiento con OZE: el porcentaje de tinción cuando se utilizó OZE fue mayor que cuando se utilizó MTA o IV. No se encontraron diferencias significativas entre el tratamiento con OZE y el control positivo. El control negativo difirió significativamente del resto de los tratamientos: el porcentaje de tinción fue menor en el control negativo.

\begin{tabular}{|c|c|c|c|c|c|c|c|c|c|}
\hline \multirow[t]{2}{*}{ Tratamiento } & \multicolumn{8}{|c|}{ Porcentaje de tinción } & \multirow{2}{*}{$\begin{array}{c}\text { Prueba de Kruskal-Wallis } \\
(H=52.09 ; \mathrm{g}=4 ; \mathrm{p}<0.0001) \\
\text { Comparaciones } \\
\text { de a pares post hoc }\end{array}$} \\
\hline & $n$ & Media & D.E. & Min. & Máx. & Mediana & Q1 & $\overline{Q 3}$ & \\
\hline C. positivo & $\overline{20}$ & 78.48 & 8.50 & 58.09 & 87.88 & 79.86 & 69.31 & 84.54 & $\bar{c}$ \\
\hline C. negativo & 20 & 3.55 & 7.90 & 0.00 & 25.69 & 0.00 & 0.00 & 2.02 & a \\
\hline MTA & 20 & 30.67 & 35.99 & 0.00 & 96.38 & 11.56 & 3.65 & 41.55 & $b$ \\
\hline OZE & 20 & 60.60 & 32.35 & 16.63 & 97.81 & 75.33 & 24.31 & 92.51 & $c$ \\
\hline IV & 20 & 30.23 & 32.07 & 0.00 & 80.77 & 15.01 & 3.39 & 64.82 & b \\
\hline
\end{tabular}

Tabla 1. Porcentaje de tinción en los cinco tratamientos (C. positivo: control positivo; C. negativo: control negativo; MTA: retroobturado con MTA; OZE: retroobturado con óxido de zinc eugenol; IV: retroobturado con ionómero vitreo). Prueba de Kruskal-Wallis, comparaciones de a pares: grupos sin ninguna letra común presentan diferencias significativas en el porcentaje de tinción $(\mathrm{p}<0.05)$.

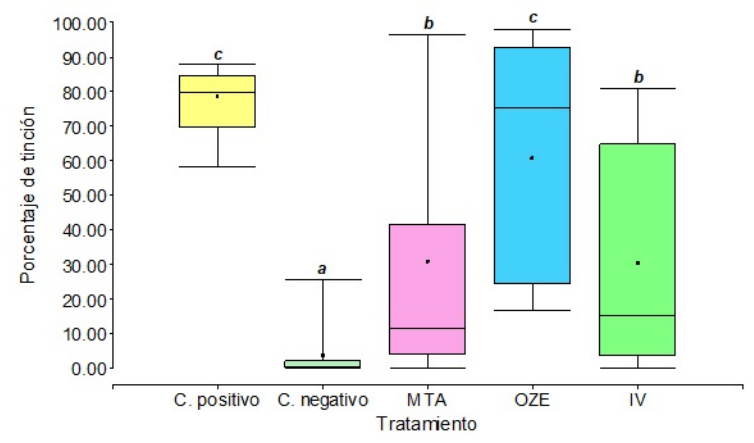

Fig. 2. Porcentaje de tinción en los cinco tratamientos. Se analizaron con la prueba no parametrica de Kruskal-Wallis, seguida por comparaciones de a pares: grupos sin ninguna letra común presentan diferencias significativas en el filtrado $(\mathrm{p}<0,05)$.

\section{DISCUSIÓN}

El control negativo fue realizado para avalar el modelo experimental. Como era esperado, este grupo presentó una microfiltración insignificante, ya que el ápice fue recubierto con dos capas de esmalte de uñas. De hecho, los tres materiales experimentales mostraron un porcentaje de filtración significativamente mayor al del control negativo. Estos resultados concuerdan con los obtenidos por Virat y col. (2). Cabe mencionar que la metodología utilizada en el presente estudio fue basada en trabajos previos de diversos autores $^{22,23,26,30,31}$ junto con la tinción apical con de azul de metileno al $2 \%$.

La elección de azul de metileno al $2 \%$ para evaluación de microfiltración se da por su rentabilidad y fácil manipulación. El azul de metileno elegido ya que su tamaño molecular es análogo a un subproducto bacteriano, es decir, ácido butírico, que se dice que se escapa de los conductos radiculares enfermos, lo que lleva a irritación periapical $^{16,32,33}$ )

Torabinejad y col. en 1995 demostraron que el seccionamiento de tipo longitudinal puede crear orificios falsos en la interfase entre la dentina y el material de relleno apical, que pueden afectar la evaluación de la adaptación marginal. ${ }^{34}$ Para evitar esta situación, se indica realizar secciones transversales que permiten la visualización de la interfaz de restauración-dentina a lo largo de la circunferencia. ${ }^{4,32}$

El MTA ha sido un pilar de la endodoncia clínica desde su introducción a mediados de la década de 1990. Fue el primer material de restauración con capacidad para estimular el crecimiento excesivo de cemento y la formación de hueso, y también facilitar la regeneración del ligamento periodontal. Actualmente, se acepta como material de referencia para la retroobturación apical. ${ }^{35}$ Además, el MTA posee otras propiedades beneficiosas, tales como su biocompatibilidad. ${ }^{21}$ El MTA es un excelente material para retroopturación apical, pero también debemos de tener en cuenta otros biomateriales como Bioaggregate, Biodentine, Endosecuence y CEM, ya que se ha demostrado que aportan buenos resultados y pueden servir como alternativa al MTA.

Los cementos de ionómero de vidrio poseen ciertas propiedades únicas que los hacen útiles como materiales restauradores, incluida la adhesión a la estructura del diente, lo que potencialmente reduce la microfiltración, y la biocompatibilidad. La actividad antibacteriana puede deberse al bajo $\mathrm{pH}$ de los cementos antes del fraguado y su capacidad para liberar fluoruro. Según las composiciones tienen diferentes tipos de resistencia y durabilidad. ${ }^{36}$

Se han realizado varios estudios in vitro utilizando IV como material de obturación retrograda, comparando su efectividad con diferentes materiales. El IV resultó ser el material que permite el mejor sellado según los trabajos de Sutimantanakul y col. en $2000^{28}$ y de otros. ${ }^{37,38}$ Sin embargo, estos estudios presentan limitaciones y los resultados son contradictorios según los grupos de estudio de Bruyne y De 
Moor en 2004. ${ }^{39}$

Según Reddy NV en 2019 el cemento Portland proporciona un sello efectivo para las perforaciones furcales de los dientes primarios y puede considerarse un sustituto más económico de la MTA como material de reparación que mejora el pronóstico de los dientes primarios perforados que de otro modo se extraerían. ${ }^{40}$

El ambiente húmedo no parece ser perjudicial para la superficie de retroobturación apical y los IV utilizados en esta técnica parecen ser menos susceptibles a la humedad de lo esperado, tal como ha sido demostrado in vitro e in vivo. $^{41,42}$

Según Khan y Abidi en 2018 quienes también compararon MTA con IV no obtuvieron diferencias significativas en la permeabilidad del colorante in vitro por lo que concluyeron que el MTA es equivalente al IV en la prevención de microfiltración cuando se usa como material de retrobturación. ${ }^{43}$

\section{CONCLUSIÓN}

Se puede concluir que el IV y el MTA tienen una capacidad de sellado similar en la retroobturación apical in vitro. A su vez, en iguales condiciones, el OZE tiene una dudosa capacidad de sellado. El presente estudio otorga elementos al odontólogo que contribuyen a la decisión respecto al material de obturación a emplear, según su capacidad de sellado. Sin embargo, no contempla otras propiedades inherentes a los materiales que hay que tener en cuenta al momento de seleccionar el más adecuado para una cirugía apical. No solo es importante evaluar todos los aspectos inherentes al material, sino también considerar su interacción con los tejidos. Si bien el MTA y el IV presentaron similares resultados en cuanto a su capacidad de sellado in vitro, deberían ser evaluados in vivo, teniendo en cuenta que hay múltiples factores que podrían modificar su comportamiento. Tales estudios deberían apuntar a una evaluación clínica y a largo plazo, de las propiedades observadas in vitro. Actualmente son de elección nuevos materiales específicos en las técnicas de retroobturacion apical. Pero no deja de estar en vigencia el uso clínico de IV en las cirugías apicales por lo que es importante recordar las características que debe tener el mismo y las indicaciones de manipulación pudiendo favorecer en el pronóstico del tratamiento.

\section{Referencias}

1 John Ide Ingle, Glickman GN, Hartwell GR. Ingle's Endodontics, chapter 23: Endodontic surgery. Ontario, Canada, Bc Decker Inc., 6th edition. 2008. p.1235
2 Virat G, Anjali S, Inder K. P, Ramandeep Singh G.Comparative evaluation of microleakage of various retrograde filling materials: An in vitro study. J Nat Sci Biol Med. 2013 Jul-Dec; 4(2): 403-408.

3 Majid K, Mohammad JE, Saeed A. Comparison of Bacterial and Dye Microleakage of Different Root-End Filling Materials. Iran Endod J. 2010 Winter; 5(1): 17-22.

4 Gartner AH, Dom SO. Advances in endodontic surgery. Dental Clinics of North America. 1992; 357-78.

5 De Bruyne MA, De Moor RJ. Long-Term sealing ability of resilon apical root-end fillings. Int endod J. 2009;42 (10): 884-92.

6 Torabinejad M, Pitt Ford TR. Root end filling materials: a review. Endod Dent Traumatol 1996;12:161-78.

7 Szeremeta-Brower TL, VanCura JE, Zaki AE. A comparison of the sealing properties of different retrograde techniques: an autoradiographic study. Oral Surg Oral Med oral Pathol 1985;59:82-7.

8 Pitt Ford TR, Andreasen JO, Dorn SO, Kariyawasam SP. Effect of Super EBA as a root-end filling on healing after replantation. J Endod 1995;21:13-5.

9 Torabinejad M, Hong CU, Pitt Ford TR, Kettering JD. Cytotoxicity of four root end filling materials. J Endod 1995; 21:489-92.

10 Torabinejad M, Chivian N. Clinical applications of mineral trioxide aggregate. J Endod. Loma Linda University 1999;25(3):197-205.

11 Torabinejad M, Pitt Ford TR, McKendry DJ, Abedi HR, Miller DA, Kariyawasam SP. Histologic assessment of mineral trioxide aggregate as a root-end filling in monkeys. J Endod 1997; 23:225-8.

12 Baek SH, Plenk H Jr, Kim S. Periapical tissue responses and cementum regeneration with amalgam, SuperEBA, and MTA as root-end filling materials. J Endod 2005;31:444 -9.

13 Shin S. In vitro studies addressing cellular mechanisms underlying the bone and dentin inductive property of mineral trioxide aggregate. Master thesis in oral biology. (Thesis) University of Pennsylvania, 2004.

14 Torabinejad M, Hong CU, Pitt Ford TR, Kaiyawasam SP. Tissue reaction to implanted super-EBA and mineral trioxide aggregate in the mandible of guinea pigs: a preliminary report. J Endod 1995; 21:569 -71.

15 Chong BS, Pitt Ford TR, Hudson MB. A prospective clinical study of Mineral Trioxide Aggregate and IRM when used as root-end filling materials in endodontic surgery. Int Endod J 2003;36:520-6.

16 Lone MM, Khan FR, Lone MA. Evaluation of Microleakage in Single-Rooted Teeth Obturated with Thermoplasticized Gutta-Percha Using Various Endodontic Sealers: An In-Vitro Study. J Coll Physicians Surg Pak. 2018 May;28(5):339-343.

17 Lone MM, Khan FR. Evaluation Of Micro Leakage Of Root Canals Filled With Different Obturation Techniques: An In Vitro Study. J Ayub Med Coll Abbottabad. 2018 Jan- 
Mar;30(1):35-39.

18 Limkangwalmongkol S, Abbott PV, Sandler AB. Apical dye 33 penetration with four root canal sealers and gutta-percha using longitudinal sectioning. J Endod. 1992 Nov;18(11): 535-9

19 Aqrabawi J. Sealing ability of amalgam, Super EBA cement, and MTA when used as retrograde filling materials. Braz Dent J 2000;188:266-268.

20 Kim S, Pecora G, Rubinstein R. Comparison of traditional 35 Parirokh M, Torabinejad M. Mineral trioxide aggregate: A and microsurgery in endodontics. In: Kim S, Pecora G, Rubinstein R, eds. Color atlas of microsurgery in endodontics. Philadelphia: W.B. Saunders, 2001:5-11.

21 Torabinejad M, Hong CU, Pitt Ford TR. Physical properties of a new root end filling material. J Endodon; 1995 21: 34953

22 Ahlberg KM, Assavanop P, Tay WM. A comparison of the apical dye penetration patterns shown by methylene blue 37 and india ink in root-fillied teeth. Int Endod J. 1995 jan; 28 (1): $30-4$.

23 Vogt BF, Xavier CB, Demarco FF, Padilha MS. Dentin penetrability evaluation of three different dyes in root-end cavities filled with mineral trioxide aggregate (MTA). Braz 39 Dent J 2000;188:266-268.

24 Tanomaru Filho M, Faleiros FB, Silva GF. Sealing ability of retrograde obturation materials containing calcium hydroxi- 40 de or MTA. Acta Odontol. Latinoam. 2011;24(1):110-114

25 Di Rienzo J.A., Casanoves F., Balzarini M.G., Gonzalez L., Tablada M., Robledo C.W. InfoStat versión 2013p, Grupo InfoStat, FCA, Universidad Nacional de Córdoba, Argenti- 41 na. 2013. URL http://www.infostat.com.ar.

26 Pereira CL, Cenci MS, Demarco FF. Sealing ability of MTA, Super-EBA, Vitremer and amalgam as root-end filling materials. Braz Oral Res 2004;18:317-321.

27 Adamo HL, Buruiana R, Schertzer L, Boylan RJ. A comparison of MTA, Super-EBA, composite and amalgam as rootend filling materials using a bacterial microleakage model. Int Endod J. 1999;32:197-203

28 Sutimantanakul S., Worayoskowit W, Mangkirnkarn C. Retrograde seal in ultrasonically prepared canals. J Endod. 2000 Aug;26(8):444-6.

29 Chong BS, Pitt Ford TR, Watson TF, Wilson RF. Sealing ability of potential retrograde root filling materials. Endod Dent Traumatol. 1995;11:264-9

30 Post, Letícia; Lima, Fábio; Xavier, Cristina; Demarco, Flavio; Gerhardt-Oliveira, Marília. Sealing ability of MTA and amalgam in different root-end preparations and resection bevel angles: An in vitro evaluation using marginal dye leakage. Brazilian dental journal. 2010 21. 416-9.

31 Xavier CB, Weismann R, Oliveira MG, Demarco FF, Pozza DH. Root-end filling materials: apical microleakage and marginal adaptation. J Endod 2005;31:539-542.

32 Punia SK, Nadig P, Punia V. An in vitro assessment of apical microleakage in root canals obturated with gutta-flow, resilon, thermafil and lateral condensation: A stereomicroscopic study. J Conserv Dent 2011; 14:173-7.

Bodrumlu E, Tunga U. Apical leakage of Resilon obturation material. J Contemp Dent Pract 2006; 7:45-52. J Coll Physicians Surg Pak. 2018 May;28(5):339-343.

34 Torabinejad M, Smith PW, Kettering JD, Pitt Ford TR. Comparative investigation of marginal adaptation of mineral trioxide aggregate and other commonly used root-end filling materials. J Endod. 1995 Jun;21(6):295-9.

comprehensive literature review - Part I: Chemical, physical, and antibacterial properties. J Endod. 2010;36:16-27.

36 Moshaverinia M, Navas A, Jahedmanesh N, Shah KC, Moshaverinia A, Ansari S. Comparative evaluation of the physical properties of a reinforced glass ionomer dental restorative material. J Prosthet Dent. 2019 Aug;122(2):154159.

37 Wu MK, Kontakiotis EG Long-term seal provided by some root-end filling material. J Endod. 1998 Aug;24(8):557-60.

38 Gerhards F, Wagner W. Sealing ability of five different retrograde filling materials. Journal of endodontics. 1996 22, 463-6.

39 Bruyne MA, De Moor RJ., The use of glass ionomer cements in both conventional and surgical endodontics. Int Endod J. 2004 Feb;37(2):91-104.

40 Reddy NV, Srujana P, et al. Sealing Ability of MTA vs Portland Cement in the Repair of Furcal Perforations of Primary Molars: A Dye Extraction Leakage Model-An In Vitro Study. Int J Clin Pediatr Dent 2019;12(2):83-87.

41 Jesslén P, Zetterqvist L, Heimdahl A. Long-term results of amalgam versus glass ionomer cement as apical sealant after apicectomy. Oral Surg Oral Med Oral Pathol Oral Radiol Endod. 1995 Jan;79(1):101-3

42 Friedman S, Rotstein I, Mahamid A., In vivo efficacy of various retrofills and of $\mathrm{CO} 2$ laser in apical surgery, Endod Dent Traumatol. 1991 Feb;7(1):19-25.

43 Khan TN, Abidi SYA. Comparison of Retrograde, Primary and Secondary Bonding Materials with Tooth Substance J Coll Physicians Surg Pak. 2018 Jan;28(1):9-12.

Recibido: 01 de Noviembre del 2019

Aceptado: 15 de Diciembre del 2019 